\section{Impact of a regional anesthesia analgesia pro- gram for outpatient foot surgery}

Linda Collins MB BCH BAO FFARCSI, Abdulla Halwani MD, Himat Vaghadia BSC MB BS MHSC FRCA FRCPC*

Purpose: To examine the effects of a Regional Anesthesia and Analgesia (RAA) program for outpatient foot surgery in an outpatient unit.

Methods: The RAA program was implemented in our Surgical Day Care Center (SDCC) in 1992-93. Fifty charts were randomly selected for each of two periods, 1990 (pre-program) and 1995 (post-program). One surgeon operated on all patients. Preoperative, intraoperative and postoperative information collected included demographic data, block type, analgesic requirements, nursing visits and complications. A time analysis of the perioperative course was also performed.

Results: The use of regional block increased from $2 \%$ to $82 \%$ as a result of the RAA program. This was not accompanied by a change in operating room efficiency (anesthetic time, preparation time, exit time). Patient stay in the PACU and discharge lounge was reduced by a mean difference of $7 \mathrm{~min}$ (Upper $95 \% \mathrm{Cl}=24 \mathrm{~min}$ ) and fentanyl consumption in the OR was reduced by $35 \mu \mathrm{g}(95 \% \mathrm{Cl}=-8,-62 \mu \mathrm{g})$. The number of patients who required $>1<4$ nursing visits for analgesia administration in the PACU decreased from 17 to 9 after program implementation.

Conclusions: Regional anesthesia is more likely to be offered to outpatients if it is introduced as a formal RAA program. Such a program did not have a negative impact on operating room efficiency and was beneficial in reducing patient stay in the PACU and discharge lounge. Facility discharge processes may need to be modified to obtain the maximum benefits from such RAA programs.

Objectif : Examiner les effets d'un programme d'anesthésie et d'analgésie régionales (AAR) pour une intervention au pied en chirurgie ambulatoire.

Méthode : Le programme d'AAR a été mis en application à notre Centre de chirurgie d'un jour (CC)) en 199293. Cinquante cas ont été sélectionnés au hasard pour chacune des deux périodes, 1990 (préprogramme) et 1995 (postprogramme). Un seul chirurgien a procédé à toutes les interventions. Les renseignements préopératoires, peropératoires et postopératoires recueillis comprenaient des données démographiques, le type d'anesthésie régionale, les besoins analgésiques, le nombre de visites du personnel infirmier et les complications. Une analyse temporelle de l'évolution périopératoire a aussi été réalisée.

Résultats : Avec programme d'AAR, le recours à l'anesthésie régionale est passé de $2 \%$ à $82 \%$. Ce qui n'a pas amélioré l'efficacité en salle d'opération (durée de l'anesthésique, temps de préparation, moment de la sortie). Par contre, le séjour du patient à la salle de réveil et au salon de repos a été réduit de 7 min en moyenne (plus de $95 \%, I C=24 \mathrm{~min}$ ). La consommation de fentanyl dans la salle d'opération a été réduite de 35 ug $(95 \%, I C$ $=-8,-62 \mu \mathrm{g}$ ). Le nombre de patients qui ont eu besoin de $>1<4$ visites du personnel infirmier pour l'administration de l'analgésie à la salle de réveil a baissé de 17 à 9 après la mise en oeurre du programme.

Conclusion : On offre plus facilement l'anesthésie régionale en chirurgie ambulatoire lorsqu'elle fait partie d'un programme formel d'ARA. Ce programme ne nuit pas à l'efficacité de la salle d'opération et permet de réduire le séjour du patient à la salle de réveil et au salon de repos. Certaines modifications peuvent être nécessaires pour faciliter la sortie du service et obtenir les meilleurs bénéfices d'un programme d'ARA.

From the Section of Ambulatory Anesthesia, Department of Anesthesia, Vancouver General Hospital and Department of Health Care and Epidemiology, " University of British Columbia, 910 West 10th Avenue, 3rd Floor, Vancouver, B.C. V5Z 4E3 Canada. Address correspondence to: Dr Himat Vaghadia, Department of Anesthesia, 910 west 10th Ave, $3^{\text {rd }}$ Floor, Vancouver, BC V5Z 4E3

Canada. Phone: 604-875-4575; Fax: 604-875-5209; E-mail: hvaghadi@vanhosp.bc.ca Accepted for publication May 16,1999 
OOT surgery is often performed in an Ambulatory Care setting. Ideally, the anesthetic technique most suitable for this procedure should provide rapid patient recovery, minimal nursing care requirements in the PACU and reduced hospital discharge times. The postoperative pain that follows can be moderate to severe in intensity and difficult to control with oral analgesics. ${ }^{1}$ Thus, regional anesthesia as a method for providing postoperative pain control may be advantageous in this setting. However there is a perception that the provision of regional anesthesia may increase the time required for administration of anesthesia, thereby leading to inefficiency and operating room delay. ${ }^{2}$ With the increasing focus on health care costs, anesthesiologists along with other medical care specialists are forced to become more efficient in their practice behaviour. ${ }^{3}$ One method of effecting successful and efficient change in health care is to introduce the concept as a formal program. A Regional Anesthesia Analgesia Program (RAA) for foot surgery was implemented in the Surgical Day Care Center (SDCC) at the Vancouver General Hospital in 1992-1993 to improve the quality of postoperative analgesia. The purpose of the present study was to perform a formal evaluation of this Regional Anesthesia Analgesia Program. The main outcomes of interest were utilization of regional blocks, operating room efficiency, intraoperative and postoperative analgesia, and PACU efficiency.

\section{Methods}

After obtaining institutional approval, a retrospective review of foot surgery in the surgical day Unit was performed on 100 charts. Fifty charts were selected for each of two periods, 1990 (Pre-program- Group 1) and 1995 (Post-program- Group 2) using a simple random sampling technique based on a random number table. The program was established in 1992-3 and 1995 was chosen as the post program year to allow sufficient time for 'buy in'. The Regional Anesthesia and Analgesia (RAA) program was developed after performing detailed studies on the optimal method of regional anesthesia for foot surgery in our SDCC. ${ }^{1,4}$ Patient information for program assessment was obtained using the Prism Abstracting System (Prism Hospital Software, Coquitlam, B.C., Canada). Procedures were coded using the ICD-9-CM method. ${ }^{5}$ All information was obtained from the patient charts. Parameters collected included demographic data, surgical procedure, type of regional anesthetic block performed, Operating Room and Post Anesthetic Care Unit duration, frequency of nursing visits for analgesia administration in PACU, analgesia usage in the OR and PACU and any compli- cations arising. One surgeon operated on all patients. All anesthetics were administered by certified anesthesiologists ( $n=44)$ assigned to work in the SDCC. There was no change in nurses in the PACU or discharge lounge over the years 1990-95.

The perioperative period was divided into discrete intervals: Interval A, the time from entry of the patient into the OR to the time the attending anesthesiologist started applying the monitors; Interval B, the time taken from anesthetic induction to commencing skin preparation of the surgical field; Interval $\mathrm{C}$, the time from commencing skin preparation to commencing the skin incision; Interval $\mathrm{D}$, the time from start of skin incision to skin closure; Interval E, the time from skin closure to completion of surgical dressing application; Interval F, the time from entry to exit from the PACU; Interval G, the time from entry to exit from the Discharge Lounge.

On arrival in the $\mathrm{OR}$, all patients were monitored with an ECG, pulse oximeter and an automated BP. An intravenous line previously established in the preoperative lounge was used to induce general anesthesia with propofol. Airway management consisted of a laryngeal mask or endotracheal tube at the discretion of the anesthesiologist with a muscle relaxant (vecuronium) as appropriate. General anesthesia was maintained with isoflurane in nitrous oxide and oxygen and respirations were either spontaneous or assisted. Intraoperative analgesia was provided with fentanyl and morphine. Under general anesthesia, an ankle block or lateral popliteal block was performed by the anesthesiologist according to the method described previously. ${ }^{1,4}$ Wound infiltration, when performed, was always done by the surgeon. A thigh tourniquet was then applied to all patients after exsanguination to facilitate hemostasis. At the conclusion of surgery, patients woke up, the trachea was extubated and they were transported to the PACU. In the PACU, the nursing protocol for analgesia was unchanged over time and consisted of $25 \mu \mathrm{g}$ fentanyl or 1-2 mg morphine iv 5-10 min prn.

The standard discharge criteria in our PACU are: oriented to person, time, and place; stable vital signs for $>30 \mathrm{~min}$; hemostasis of surgical wounds; absence of side effects; adequate pain control with oral analgesics ( $<3$ on verbal rating scale); able to change clothes and ambulate with crutches without assistance. Resolution of the nerve block was not required because analgesia from these blocks was expected to last for more than six hours. All time data were taken from the OR and PACU nursing records where these are entered in hours and minutes by the circulating nurse and the PACU nurse respectively. The interval 
times were obtained by subtracting the appropriate beginning and ending times and all periods were measured in minutes.

Statististical analysis was performed by the hospital Clinical Epidemiology and Research Department. All data were entered into a spreadsheet (Microsoft Excel) program and analyzed using the SAS software (SAS Institute, Cary NC). All valid observations were described with descriptive statistics using means and standard deviations, and differences between groups were determined using $95 \%$ confidence intervals. We were advised not to perform further statistical tests to generate $P$ values because we were not testing a specific hypothesis. Rather, we were examining the two samples to determine if there was a clinically significant difference. However, where the $95 \%$ confidence intervals of the difference between the means does not include zero, the difference is also statistically significant $(P<0.05)$.

\section{Results}

There was no difference between groups with respect to age, sex and weight (Table I). The surgical procedures performed are listed in Table II. There was an increase in the number of blocks performed after the implementation of the Regional Anesthesia Analgesia Program (RAA). Only four regional techniques were performed in 1990 and, of these, only $1 / 50(2 \%)$ was likely to provide prolonged postoperative analgesia. After implementation of the RAA, there was a large increase in lateral popliteal and ankle blocks so that $41 / 50(82 \%)$
TABLE I Patient demographics

\begin{tabular}{lll}
\hline & $\begin{array}{l}\text { Group I } \\
n=50\end{array}$ & $\begin{array}{l}\text { Group 2 } \\
n=50\end{array}$ \\
\hline Age $(\mathrm{yr})$ & $47(16-68)$ & $47(25-73)$ \\
Weight $(\mathrm{kg})$ & $68(46-120)$ & $69(50-107)$ \\
Sex $($ Male $/$ Female $)$ & $10 / 40$ & $10 / 40$ \\
\hline
\end{tabular}

Values: mean (range)

TABLE II Surgical procedures and Regional Anesthetic Blocks

\begin{tabular}{lll}
\hline & $\begin{array}{l}\text { Group 1 } \\
n=50\end{array}$ & $\begin{array}{l}\text { Group 2 } \\
n=50\end{array}$ \\
\hline $\begin{array}{ll}\text { Operation } \\
\text { Morton's Neuroma }\end{array}$ & 8 & 1 \\
Resection Realignment & 17 & 10 \\
Excision of Ganglion & 2 & \\
Reconstruction & 5 & 14 \\
MTP Anthrodesis & 3 & 5 \\
Chevron/Cheilectomy & 11 & 9 \\
Plantar Fasciectomy & 1 & \\
ORIF ankle & 1 & \\
ROO plate and screws & 1 & 6 \\
Ankle Arthroplasty & 1 & 2 \\
Aken Osteotomy & & 1 \\
R/O Callus & & 1 \\
Regional Anesthetic Technique & & 34 \\
Lateral Popliteal block & - & 7 \\
Ankle block & - & - \\
Wound infiltration & 1 & 1 \\
Spinal & 3 & \\
\hline
\end{tabular}

TABLE III Duration of time intervals

\begin{tabular}{|c|c|c|c|c|}
\hline & Group I & Group II & Difference & Upper $95 \% C I$ \\
\hline Interval A (Entry time) & $3 \pm 7.1$ & $1 \pm 3.3$ & -2 & 4 \\
\hline Interval B (Anesthesia time) & $12 \pm 8.6)$ & $13 \pm 7.3$ & +1 & 2 \\
\hline Interval C (Prep time) & $7 \pm 5.4$ & $7 \pm 6.7$ & & 2 \\
\hline Interval D (Surgery time) & $50 \pm 21.1$ & $49 \pm 25.5$ & -1 & 10 \\
\hline Interval E (Exit time) & $4 \pm 2.3$ & $5 \pm 3.8$ & -1 & 0.2 \\
\hline Interval F (PACU time) & $85 \pm 41$ & $78 \pm 45$ & -7 & 24 \\
\hline Interval G (Discharge lounge time) & $85 \pm 47$ & $78 \pm 38$ & -7 & 24 \\
\hline
\end{tabular}

Values: mean $\pm S, D$ min

TABLE IV Opioid Administration in the OR and PACU

\begin{tabular}{lllll}
\hline & Group 1 & Group 2 & Difference & $95 \%$ CI \\
\hline Opioids in OR & & & & $-8.8,-62.0$ \\
Fentanyl $\mu \mathrm{g}$ & $119.3 \pm 56.6$ & $83.9 \pm 36.8$ & -35.4 & \\
$\quad$ Morphine $\mathrm{mg}$ & $3.0 \pm 0.0$ & $7.6 \pm 3.8$ & +4.6 & \\
Opioids in PACU & & & & \\
Fentanyl $\mu \mathrm{g}$ & $69.0 \pm 45.0$ & $81.0 \pm 36.0$ & & \\
Morphine $\mathrm{mg}$ & $7.6 \pm 4.8$ & $7.3 \pm 8.6$ & & \\
\hline
\end{tabular}


of patients had some form of regional analgesia likely to last into the postoperative period (Table II).

The surgery times were similar in both groups. Implementation of a RAA program did not increase the entry time, anesthetic time, surgical preparation or exit time (Table III). The mean time required for administration of anesthesia in 1990 was $12 \mathrm{~min}( \pm$ 8.6) compared with $13 \mathrm{~min}( \pm 7.3)$ in 1995. The duration of stay in the PACU and Discharge Lounge were reduced in the post RAA group by seven minutes. However, since the upper $95 \%$ confidence intervals were as large as $24 \mathrm{~min}$, these differences are clinically important. There was a trend towards more nursing visits to administer analgesia in the PACU for patients anesthetized in 1990 (Group 1) compared with those anesthetized in 1995 (Group 2) (Figure). The number of patients who required $>1<4$ nursing visits for analgesia administration in the PACU decreased from 17 to 9 after program implementation. Although this did not reach statistical significance the magnitude of this change may be clinically significant. The surgeon also stated that pain related post operative complaints from patients were completely eliminated since program implementation.

The mean amount of fentanyl administered in the $\mathrm{OR}$ was higher in Group 1 than in Group 2. Other differences in opioid administration in the $O R$ and PACU were not statistically significant (Table IV). There were no complications reported with the regional anesthetic blocks. A total of three differences of clinical and statistical significance were found between the two groups.

\section{Discussion}

In an attempt to assess the effect of implementing a Regional Anesthesia Analgesia Program (RAA), we decided to study the charts of 100 patients undergoing foot surgery in the Surgical Day Unit in the years 1990 (pre program) and 1995 (post program) retrospectively. The RAA program was successful in increasing the utilization of regional blocks from $2 \%$ to $82 \%$. This increase in regional blocks for foot surgery was not accompanied by any change in OR efficiency (anesthetic time, preparation time, exit time). However, patient stay in the PACU and discharge lounge decreased after implementation of the RAA program. Opioid consumption in the OR also decreased after program implementation.

The RAA program was established to improve the quality of postoperative analgesia in-patients undergoing outpatient foot surgery. The program was developed upon the request of our surgeon who noticed an increase in complaints from patients due to excessive

\section{No. of Nursing Visits in PACU}

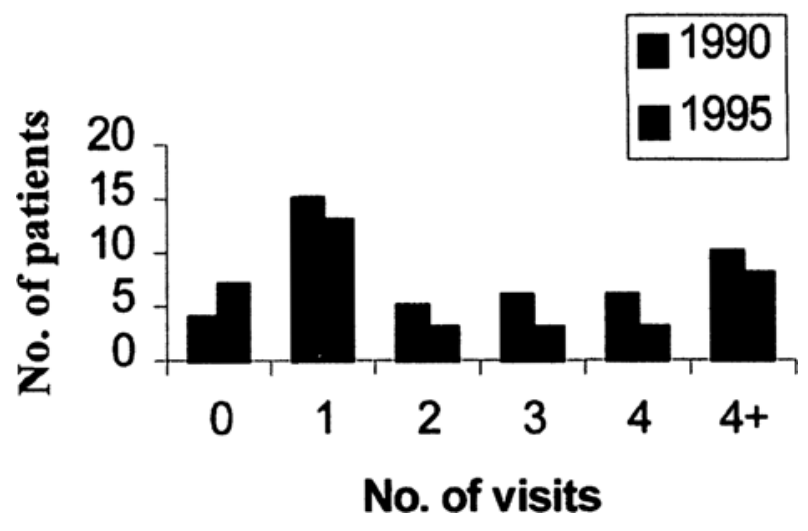

FIGURE

pain at home. Pain after orthopedic procedures can account for a readmission rate as high as $\mathbf{5 0 \%}$, and this rate can be reduced by the use of long acting local anesthetic nerve blocks. ${ }^{\circ}$ The problem was systematically studied in our SDCC and an appropriate type of nerve block was developed. ${ }^{1,4}$ The program was introduced after in-servicing of anesthesiologists (rounds and instruction in the OR) and nursing staff (rounds). In addition, the surgeon was also taught how to perform the ankle and lateral popliteal blocks. When the staff anesthesiologist was not comfortable in performing the blocks, the surgeon performed the block. In the event that the surgeon was unsuccessful (unable to elicit a twitch with a nerve stimulator for the lateral popliteal block), wound infiltration was performed at the time of wound closure. Since all patients required general anesthesia to tolerate a thigh tourniquet, efficacy of the block was only evident in the recovery room when the patient emerged from GA. In a retrospective study such as this, there was no clear way to assess block efficacy from the nursing notes other than to determine the amount of analgesics consumed in PACU. Once the program was implemented, we estimated that at least one year would be required for 'buy in'. Program evaluation was, therefore, performed well after this 'buy in' period. The purpose of this study was to evaluate the effectiveness of the program in addressing the stated problem and to determine its impact on overall efficiency in our SDCC. The design methodology chosen for program evaluation was that of pretest-posttest design.? Although this method is acceptable for program evaluation it has some disadvantages. Important 
problems are: a) it is not possible to eliminate any real effect of the program from other simultaneously occurring factors, $b$ ) regression to mean may account for the pretest-posttest differences, c) the effects of participation in a program may have an effect on program performance alone, and, d) expectations of the observer assessing the program may also have an effect. However, there were no other simultaneous changes made in the care of patients in our SDCC. It is also unlikely that regression to mean contributed to our findings because we have previously demonstrated an effect with randomized studies. ${ }^{1,4}$ The risks of sensitization are probably insignificant because, at the time of program implementation, we did not inform any of the medical and nursing staff of our intention to evaluate the program at a subsequent date. Finally, utilizing a random methodology in chart selection also minimized reactiveness.

Currently, there are concerns that outpatients do not have adequate access to regional anesthesia. ${ }^{8}$ In a large-scale epidemiological study of four major teaching hospitals, it was demonstrated that less than $3 \%$ of outpatients were managed with regional techniques. ${ }^{9}$ An important factor that may account for this low utilization of regional anesthesia in outpatients may be perceptions of less time efficiency. ${ }^{8}$ Our data show that such fears are not valid. Our RAA program had no impact on OR efficiency. Recently, it has been demonstrated that foot surgery with metatarsal or ankle block and sedation was not associated with any decrease in OR efficiency. ${ }^{10}$ Our experience also confirms that for regional anesthesia to be successful, it has to be established as the norm for the institution or for a particular surgical group. ${ }^{2}$

The potential benefits of regional analgesia in the postoperative period are also little appreciated by surgeons. Surgeons do not spend enough time in the PACU or discharge lounge to witness the benefits on pain management and efficiency. Our program decreased duration of PACU and discharge lounge stay considerably. The differences were clinically significant (upper $95 \% \mathrm{CI}=24 \mathrm{~min}$ ) and could have a major impact on overall efficiency, resource planning and staffing of outpatient facilities. A $24 \mathrm{~min}$ delay for some patients could make a difference in terms of unnecessary cancellation in the OR; unnecessary admission and delay in patient pick up - this study was not designed to examine such issues. We also observed a trend towards fewer nursing visits for the administration of analgesics in the PACU once the RAA program was implemented. The number of patients who required $>1<4$ nursing visits for analgesia administration in the PACU decreased from 17 to 9 after program implementation. Although this did not reach statistical significance the magnitude of this change may be clinically significant. One reason for not achieving statistical significance is that the nursing protocol for postoperative management was not changed after implementing the RAA program. Thus, many patients received routine oral analgesia in the PACU to prepare them for the ride home, rather than for actual pain. In fact, VAS scores for pain at discharge from the SDCC were no different from baseline values before surgery with the use of block techniques used with our RAA program. ${ }^{3,4}$ Our findings of shorter recovery times are also confirmed by other studies on outpatient foot surgery ${ }^{10}$ and arthroscopy ${ }^{11}$ that demonstrated a shorter recovery room stay and lower hospital costs with regional techniques. The superior postoperative analgesia with regional techniques is particularly important in orthopedic procedures where rates of readmission due to pain can be dramatically reduced. ${ }^{6}$ Readmission rates after a variety of orthopedic procedures are also lower with regional techniques compared with general anesthesia. ${ }^{12}$

Considerable cost savings can also be achieved if an RAA program is combined with a 'fast track' program designed to minimize hospital stay. Patients who have foot surgery under ankle block can bypass the PACU and be discharged home directly from the operating room. ${ }^{2,10}$ Our RAA program was not able to achieve this objective because all patients required light general anesthesia to tolerate a thigh tourniquet. In addition, we did not combine the RAA program with a 'fast track recovery' approach in the PACU. The results of the present investigation suggest that PACU protocols and discharge criteria in our SDCC need to be tailored more precisely in order to maximize the benefits of our RAA program. The impact of nursing management on the success of any RAA program should not be under emphasized. Recently, it has been demonstrated that postoperative nursing care was the single most important factor affecting discharge time in adult outpatients. ${ }^{13}$ As a result of this program evaluation, we have implemented some changes - all patients now receive pre-emptive analgesia (acetaminophen, $975 \mathrm{mg}, p o$ ) in the preoperative lounge. It is hoped that this will reduce 'routine' nursing visits in the PACU to provide oral analgesia as a prelude to discharge planning. In the future attention will be directed toward suitable 'fast track' processes in the PACU.

In conclusion, a Regional Anesthesia and Analgesia Program can be implemented in outpatients without any impact on operating room efficiency. Potential benefits include greater opportunity for outpatients to access regional anesthesia, shorter recovery time, improved analgesia and an ability to fast track the discharge process. 


\section{References}

1 McLeod DH, Wong DHW, Claridge RJ, Merrick PM. Lateral popliteal sciatic nerve block compared with subcutaneous infiltration for analgesia following foot surgery. Can J Anaesth 1994; 41: 673-6.

2 Greenberg CP. Practical, cost-effective regional anesthesia for ambulatory surgery. J Clin Anesth 1995; 7 : 614-21.

3 Becker KE Jr, Carrithers J. Practical methods of cost containment in anesthesia and surgery. J Clin Anesth 1994; 6: 388-99.

4 McLeod DH, Wong DHW, Vagbadia H, Claridge RJ, Merrick PM. Lateral popliteal sciatic nerve block compared with ankle block for analgesia following foot surgery. Can J Anaesth 1995; 42: 765-9.

5 International Classification of Disease, 9th revision. Clinical Modification (ICD-9-CM), Vol 3: Procedures. Ann Arbour: Commission on Professional and Hospital Activities, 1968.

6 Kinnard P, Jarrett PEM. Orthopedic surgery. In: White PF (Ed.). Ambulatory Anesthesia and Surgery, Ist ed. Philadelphia: WB Saunders Company Ltd, 1997: 270-4.

7 Veney JE, Kaluzny $A D$. Experimental design as an evaluation strategy. In: Veney JE, Kaluzny AD (Eds.). Evaluation and Decision Making for Health Services Programs, 1st ed. New Jersey: Prentice Hall, 1984: 175-98.

8 Vaghadia $H$. Spinal anaesthesia for outpatients: controversies and new techniques. Can J Anaesth 1998; 45: R64-70.

9 Duncan PG, Coben MM, Tweed WA, et al. The Canadian four-centre study of anaestheric outcomes: III. Are anaesthetic complications predictable in day surgical patients? Can J Anaesth 1992; 39: 440-8.

10 Reinbart DJ, Wang WP. Cost and resource utilization for foot surgery: general vs MAC anesthesia. Anesth Analg 1996; 82: S376.

11 Patel NJ, Flasbburg MH, Paskin S, Grossman R. A regional anesthetic technique compared to general anesthesia for outpatient knee arthroscopy. Anesth Analg 1986; 65: 185-7.

12 Mingus ML. Recovery advantages of regional anesthesia compared with general anesthesia: adult patients. J Clin Anesth 1995; 7: 628-33.

13 Pavlin DJ, Rapp SE, Polissar NL, Malmgren JA, Koerschgen $M$, Keyes $H$. Factors affecting discharge time in adult outpatients. Anesth Analg 1998; 87: 816-26. 